Enfoques. Revista de Investigación en Ciencias de la Administración http://doi.org/10.33996/revistaenfoques.v4i16.96 No. 16 | Volumen 4 | Octubre - Diciembre 2020 http://revistaenfoques.org ISSN: $2616-8219$

\title{
ACTIVIDADES DE APOYO DE LA GESTIÓN LOGÍSTICA EN LAS EMPRESAS COMERCIALIZADORAS DE BEBIDAS
}

ISSN-L: $2616-8219$

pp. $239-247$

\author{
SUPPORT ACTIVITIES FOR LOGISTICS MANAGEMENT IN BEVERAGE \\ MARKETING COMPANIES
}

ATIVIDADES DE APOIO À GESTÃO LOGÍSTICA EM EMPRESAS DE
MARKETING DE BEBIDAS

Juan Carlos León

Artículo recibido julio 2020 | Arbitrado agosto 2020 | Publicado 01 de octubre 2020

\begin{abstract}
Resumen
Conociendo que el área logística es de gran apoyo en la competitividad y reducción de costos en las empresas comercializadoras de bebidas, se planteó el análisis de las actividades de apoyo en la gestión logística de las mismas. La metodología se centró en el tipo descriptiva, bajo un diseño no experimental, de campo y transeccional. Se consideró como población las cuatro empresas comercializadoras de bebidas en la Costa Oriental del Lago. Se empleó la encuesta para la recolección de datos, mediante un cuestionario, conformado por 15 ítems con escala de frecuencia. La validez se realizó a través del juicio de expertos, y para calcular su confiabilidad se empleó el Coeficiente Alfa de Cronbach, obteniéndose 0,93. El análisis de los datos se realizó mediante el análisis de la media aritmética. Se determinó muy alto cumplimiento de todas las actividades medidas: compras, embalaje, almacenaje, planificación de productos y tratamiento de mercancías.
\end{abstract}

Palabras clave: Actividades de apoyo, almacenaje; compras; embalaje; gestión logística; planificación de productos, tratamiento de mercancías

\begin{abstract}
Knowing that the logistics area is highly supportive in competitiveness and cost reduction in beverage trading companies, the analysis of support activities in their logistics management was proposed. The methodology focused on the descriptive type, under a non-experimental, field and transectional design. The four beverage trading companies on the Eastern Lake Coast were considered as population. The survey was used to collect data, using a questionnaire, made up of 15 items with a frequency scale. Validity was carried out through expert judgment, and the Cronbach Alpha Coefficient was used to calculate its reliability, obtaining 0.93. Data analysis was performed by analyzing the arithmetic mean. Very high compliance with all the measured activities was determined: purchasing, packaging, storage, and product planning and merchandise treatment.
\end{abstract}

Key words: Support activities; warehousing; purchasing; packaging; logistics management; product planning, merchandise treatment

\section{Juan Carlos León}

jcleon30@hotmail.com

Orcid: 0000-0002-0517-6246

Universidad del Zulia. Núcleo Costa Oriental del Lago. Venezuela.

Ingeniero en Mantenimiento, Mención: Mecánico, egresado de la Universidad Experimental Rafael María Baralt, Cabimas-Venezuela. Magíster Scientiarum en Gerencia de Empresas. Mención: Gerencia de Operaciones. Universidad del Zulia, Núcleo Costa Oriental del lago. Venezuela. 


\section{Resumo}

Sabendo que a área de logística é de grande suporte na competitividade e redução de custos nas empresas de marketing de bebidas, foi proposta a análise das atividades de apoio na gestão logística. A metodologia centrou-se no tipo descritivo, sob um desenho não experimental, de campo e transversal. As quatro empresas de marketing de bebidas na Costa Leste do Lago foram consideradas como população. A pesquisa foi utilizada para a coleta de dados, por meio de um questionário, composto por 15 itens com uma escala de frequência. A validade foi realizada por meio de julgamento de especialistas, e o coeficiente alfa de Cronbach foi utilizado para calcular sua confiabilidade, obtendo-se 0,93. A análise dos dados foi realizada por meio da análise da média aritmética. A conformidade muito alta foi determinada para todas as atividades medidas: compra, embalagem, armazenamento, planejamento de produto e tratamento de mercadoria.

Palavras-chave: Atividades de suporte; armazenamento; compras; embalagem; Gestão de logística; planejamento de produto, manuseio de mercadorias

\section{INTRODUCCIÓN}

Hoy día, es posible asegurar que aplicar la logística, desde el punto de vista integral, a largo plazo, es el único camino para mantener una competitividad continua de las organizaciones en el mercado, disminuyendo a su vez la inversión global en el stock con el consiguiente aumento de la rentabilidad de las diferentes unidades operativas; por ello se requiere mostrar la esencia de la gestión logística, como soporte que dota a la dirección de un mejor control del esfuerzo operativo global, al mismo tiempo que crea valor para la empresa, los proveedores y los clientes.

Concretando las ideas, según Ballou (2004, p.45) la gestión logística tiene un ámbito de alcance, por lo cual la define como: "la parte del proceso de la cadena de suministro que planea, organiza, ejecuta y controla el flujo y almacenamiento eficiente $y$ efectivos de bienes $y$ servicios, así como la información relacionada, desde el punto de origen hasta el consumo, con el fin de satisfacer los requerimientos de los clientes".

En esta perspectiva, según La Cruz (2010), en las pequeñas y medianas empresas venezolanas, posiblemente existen carencias de una visión global de la gestión logística, pues no se realizan análisis del entorno ni de las metas programadas para la distribución; tampoco se ha reconocido a esta como un factor crucial, que conduce a una verdadera ventaja competitiva, no solo por su repercusión en la satisfacción de los clientes, sino también, por la disminución de los costos asociados a los flujos tanto de materiales como de información.

En estas empresas, tal vez la logística no se toma como un sistema, sino como islas, existiendo una separación entre la logística de producción, la de abastecimiento y la de distribución, todo esto es debido al escaso conocimiento de la logística integral como herramienta para un óptimo desempeño organizacional.

Ahora bien, en el caso de la región zuliana, debido a su ubicación en el territorio nacional, alejada del centro del país, cuenta con costos logísticos elevados, los que corresponden principalmente al transporte de materiales desde dicha región, en el caso de las empresas comercializadoras de bebidas, para el traslado de las materias primas provenientes del centro y centro occidente del país.

Por ello necesariamente se debe reducir al mínimo posible los costos logísticos en la región, estando al tanto que una buena 
logística puede no solo reducir costos, sino también ayudar a incrementar las ventas, además el no llevar la misma de una manera organizada, lejos de reducir costos, los incrementa, llevando a la pérdida de tiempo, perdidas económicas y además de la pérdida de clientes.

Siendo más específicos, las empresas comercializadoras de bebidas pertenecientes a la Costa Oriental del Lago, a juicio del investigador por ser parte activa del sector, mantienen la visión de reducir los costos, en lo que respecta a la comercialización de sus productos, al mismo tiempo desligarse en ese punto neurálgico de terceros, y así poseer una autonomía en la producción de sus bebidas, siendo ellas mismas las proveedoras de sus insumos.

Sin embargo, debido a su naturaleza, y tener un crecimiento continuo, estas empresas posiblemente carecen de formalidad en sus procesos, aunado a que deben enfrentar el problema de mantener elevados inventarios de materia prima, incurriendo en altos costos, esto es debido a la incertidumbre creciente de la disponibilidad de materias primas y la asignación de divisas, costos de materias primas variantes, entre otros.

Así las cosas, las empresas comercializadoras de bebidas de esta región, deben contar con un eficiente manejo en el flujo de sus materias primas, desde el momento del pronóstico de la demanda, para gestionar el aprovisionamiento de materia prima, hasta la recepción de la misma en planta, considerando además el producto en proceso, así como el terminado, incluyendo el flujo de insumos como repuestos y otros materiales, aunado al flujo de la información, puesto que las mismas se encuentran día a día, en la búsqueda de la optimización de operaciones, con miras al aumento de su rentabilidad.
Por lo antes expuesto, fue necesario analizar su gestión logística, utilizando indicadores que ayudaran a visualizar la situación presente en las mismas, además, de esta manera atacar los problemas a tiempo y emprender el camino de la mejora continua. Debido a lo antes expuesto, se planteó en este estudio la necesidad de analizar las actividades de apoyo de la gestión logística en las empresas comercializadoras de bebidas de la Costa Oriental del Lago.

\section{MÉTODO}

Como parte del trabajo de investigación, se incluye su tipificación o clasificación, considerando el método a utilizar y el alcance del estudio a ser realizado, lo cual permitirá determinar los lineamientos de acción a establecer para dar cumplimiento a los fines del mismo. En este sentido, la investigación se centró en el tipo descriptiva, bajo un diseño no experimental, de campo y transeccional.

Se consideró como población las cuatro empresas comercializadoras de bebidas en la Costa Oriental del Lago, donde la unidad de información seleccionada fue el personal gerencial y supervisorio involucrado en las actividades logísticas.

Se empleó la encuesta para la recolección de datos, mediante un cuestionario, conformado por 15 ítems, bajo una escala de frecuencia, la cual, contiene cinco opciones dadas por las siguientes alternativas: nunca (1), casi nunca (2), algunas veces (3), casi siempre (4) y siempre (5). La validez se realizó a través del juicio de cinco expertos, y para calcular su confiabilidad se empleó el Coeficiente Alfa de Cronbach, obteniéndose 0,93 , lo cual indicó muy alta confiabilidad del mismo, siendo apto para su aplicación. 
El análisis de los datos se realizó mediante el análisis de la media aritmética. Para tal fin, el investigador diseñó un baremo, sobre la base de la puntuación más alta y más baja de la escala de referencia utilizada (5-1), el mismo se muestra en la tabla 1.

Tabla 1. Baremos para la interpretación de la media aritmética

\begin{tabular}{clccl}
\hline DIMENSIÓN & ALTERNATIVA & RANGO & INTERVALO & \multicolumn{1}{c}{ CATEGORÍA } \\
\hline & Siempre & 5 & $4.21-5.00$ & Muy alto cumplimiento \\
& Casi siempre & 4 & $3.41-4.20$ & Alto cumplimiento \\
& Algunas veces & 3 & $2.61-3.40$ & Moderado cumplimiento \\
Actividades de & Casi nunca & 2 & $1.81-2.60$ & Bajo cumplimiento \\
apoyo & Nunca & 1 & $1.00-1.80$ & Muy bajo cumplimiento \\
\hline
\end{tabular}

RESULTADOS

En cuanto a la dimensión actividades de apoyo, la cual fue medida por cinco indicadores a saber: compras, embalaje, almacenaje, planificación de productos y tratamiento de mercancías, se presentan en la tabla 2 los resultados para el primer indicador denominado compras. En la misma se muestra una media de 4,54, indicando muy alto cumplimiento de la actividad.

Tabla 2. Indicador: Compras

\begin{tabular}{|c|c|c|}
\hline Ítem & $\bar{X}$ & Categoría \\
\hline $\begin{array}{l}\text { 1. El departamento de compras identifica necesidades a través de } \\
\text { requerimientos de los usuarios. }\end{array}$ & 4,56 & $\begin{array}{l}\text { Muy alto } \\
\text { cumplimiento }\end{array}$ \\
\hline $\begin{array}{l}\text { 2. Antes de realizar las compras, se realizan revisiones del consumo } \\
\text { histórico del material. }\end{array}$ & 4,31 & $\begin{array}{l}\text { Muy alto } \\
\text { cumplimiento }\end{array}$ \\
\hline $\begin{array}{l}\text { 3. Se realiza seguimiento al cumplimiento de los plazos acordados con } \\
\text { los proveedores para la entrega de los productos comprados. }\end{array}$ & 4,75 & $\begin{array}{l}\text { Muy alto } \\
\text { cumplimiento }\end{array}$ \\
\hline Total indicador: & 4,54 & $\begin{array}{l}\text { Muy alto } \\
\text { cumplimiento }\end{array}$ \\
\hline
\end{tabular}

Se observa muy alto cumplimiento de todas las actividades medidas por los reactivos, así entonces se cumple de manera muy alta el que el departamento de compras identifique necesidades a través de requerimientos de los usuarios $(4,56)$, antes de realizar las compras realizan revisiones del consumo histórico del material $(4,31)$ y realizan seguimiento al cumplimiento de los plazos acordados con los proveedores para la entrega de los productos comprados $(4,75)$.

Este resultado de muy alto cumplimiento del indicador, coincide altamente con lo que expresan Butter y Linse (2008), para quienes la compra en las compañías se compone de seis pasos claros. En el primer paso la compañía identifica una necesidad, para la cual la respuesta es la compra de un producto, y el 
paso final es la ejecución de un contrato. Los pasos intermedios construyen un proceso organizado e informado que tiene como resultado que la compañía compre el producto correcto para cubrir su necesidad de un proveedor calificado cuyo producto es el más duradero por el precio.

Asimismo, coincide altamente con lo expuesto por el investigador cuando considera que las compras, como actividad de apoyo del proceso logístico, involucra la adquisición de materias primas, suministros y componentes. Las actividades asociadas a esta parte del proceso logístico incluyen desde la detención de la necesidad, hasta la especificación de la forma en la que se recibirán los bienes o servicios.

Los datos recogidos en la tabla 3, corresponden al indicador embalaje, en ésta se aprecia una media de 4,77 implicando muy alto cumplimiento de este indicador como actividad de apoyo del proceso logístico que se analiza. Esto se verifica porque existe muy alto cumplimiento de las actividades o procesos asociados al indicador.

Tabla 3. Indicador: Embalaje

\begin{tabular}{llcc}
\hline \multicolumn{1}{c}{ Ítem } & $\overline{\boldsymbol{X}}$ & Categoría \\
\hline 4. El embalaje utilizado ayuda a vender la mercancía mediante su & 4,69 & $\begin{array}{c}\text { Muy alto } \\
\text { diseño gráfico. }\end{array}$ \\
$\begin{array}{l}\text { 5. El embalaje utilizado protege el producto de la intemperie, } \\
\text { asegurando la integridad durante el transporte. }\end{array}$ & 4,81 & $\begin{array}{c}\text { Muy alto } \\
\text { cumplimiento }\end{array}$ \\
$\begin{array}{l}\text { 6. El embalaje utilizado se considera una inversión como parte integral } \\
\text { del producto para transportarlo, logrando que llegue a su destino }\end{array}$ & 4,81 & $\begin{array}{c}\text { Muy alto } \\
\text { cumplimiento }\end{array}$ \\
\hline & Total indicador: & $\mathbf{4 , 7 7}$ & $\begin{array}{c}\text { Muy alto } \\
\text { cumplimiento }\end{array}$
\end{tabular}

Especialmente se nota que las empresas dan muy alto cumplimiento a que el embalaje utilizado: ayude a vender la mercancía mediante su diseño gráfico $(4,69)$, proteja el producto de la intemperie, asegurando la integridad durante el transporte $(4,81)$ y se considera una inversión como parte integral del producto para transportarlo, logrando que llegue a su destino en óptimas condiciones $(4,81)$.

Este indicador cumple de forma muy alta con la teoría de Pulido (2009), para quien algunas de las funciones del embalaje, según son: proteger el contenido, facilitar la manipulación, informar sobre sus condiciones de manejo, requisitos legales, composición, ingredientes, entre otras. Dentro del establecimiento comercial, el embalaje puede ayudar a vender la mercancía mediante su diseño gráfico y estructural.

Adicional a esto, también logran alta coincidencia con la posición del investigador cuando afirma que el embalaje como actividad de apoyo al proceso logístico debe cumplir con las exigencias del mercado objetivo, las características del producto y las condiciones de transporte a que se verá sometido el producto hasta llegar a manos del consumidor. El embalaje debe proteger la mercancía durante todo el proceso de transporte, 
garantizando que ésta sea entregada en condiciones óptimas.

La tabla 4, contiene los datos referidos al indicador almacenaje. En ellos se puede apreciar como la media se ubicó en 3,81 indicando alto cumplimiento. Ello se debe fundamentalmente a que cumplen de manera moderada con el hecho de que la disposición del almacén exija los menores esfuerzos para su funcionamiento $(3,13)$. Mientras que poseen muy alto cumplimiento de que su planificación del almacenaje se inserte en la planificación general para participar de sus objetivos empresariales $(4,56)$; y denotan alto cumplimiento en que las cantidades almacenadas se calculan para que los costos que originen sean mínimos $(3,75)$.

Tabla 4. Indicador: Almacenaje

\begin{tabular}{|c|c|c|c|}
\hline & Ítem & $\overline{\boldsymbol{X}}$ & Categoría \\
\hline 7. & $\begin{array}{l}\text { La planificación del almacenaje se inserta en la planificación general } \\
\text { para participar de sus objetivos empresariales. }\end{array}$ & 4,56 & $\begin{array}{l}\text { Muy alto } \\
\text { cumplimiento }\end{array}$ \\
\hline 8. & $\begin{array}{l}\text { Las cantidades almacenadas se calculan para que los costos que } \\
\text { originen sean mínimos. }\end{array}$ & 3,75 & $\begin{array}{l}\text { Alto } \\
\text { cumplimiento }\end{array}$ \\
\hline \multirow[t]{2}{*}{9.} & $\begin{array}{l}\text { La disposición del almacén exige los menores esfuerzos para su } \\
\text { funcionamiento. }\end{array}$ & 3,13 & $\begin{array}{c}\text { Moderado } \\
\text { cumplimiento }\end{array}$ \\
\hline & Total indicador: & 4,73 & $\begin{array}{l}\text { Muy alto } \\
\text { cumplimiento }\end{array}$ \\
\hline
\end{tabular}

Los resultados se ajustaron, de manera alta, a la opinión de Ballou (2004), para quien el almacenaje es la actividad de soporte a la logística que implica el resguardo de los diferentes tipos de mercancía. Es manejado a través de una política de inventario, controla físicamente y mantiene todos los artículos inventariados. Al elaborar la estrategia de almacenaje se deben definir de manera coordinada el sistema de gestión del almacén y el modelo de almacenamiento.

También validaron lo expuesto por el investigador, cuando afirma que la actividad de almacenaje brinda apoyo al proceso logístico, permitiendo el custodio de los insumos, materiales o productos semielaborados que se requieran para el proceso de transformación productivo, así como para el resguardo de los productos finales. Por ello, la eficiente gestión de la logística dependerá en alto grado de un correcto proceso de almacenaje, que garantice las condiciones requeridas para el mantenimiento de estos materiales $y$ productos en condiciones óptimas para su procesamiento o venta.

Con relación al indicador planificación de productos, la tabla 5 muestra la información recabada, observándose un valor para la media de 4,71 indicando muy alto cumplimiento. El valor obtenido en esta media, es producto del muy alto cumplimiento dado a todos los reactivos medidos; así se cumple de manera muy alta con que: la planificación de los productos apoya al procesamiento de pedidos $(4,63)$ y es clave a la hora de optimizar los procesos logísticos (4,81); así como también su objetivo es poder estimar las cantidades que demandarán los clientes $(4,75)$. 
Tabla 5. Indicador: Planificación de productos

\begin{tabular}{llcc}
\hline \multicolumn{1}{c}{ Ítem } & $\overline{\boldsymbol{X}}$ & Categoría \\
\hline $\begin{array}{l}\text { 10. La planificación de los productos apoya al procesamiento de } \\
\text { pedidos. }\end{array}$ & 4,63 & $\begin{array}{c}\text { Muy alto } \\
\text { cumplimiento }\end{array}$ \\
$\begin{array}{l}\text { 11. El objetivo de la planificación de productos es poder estimar las } \\
\text { cantidades que demandarán los clientes. }\end{array}$ & 4,75 & $\begin{array}{c}\text { Muy alto } \\
\text { cumplimiento }\end{array}$ \\
$\begin{array}{l}\text { 12. La planificación de productos es clave a la hora de optimizar los } \\
\text { procesos logísticos. }\end{array}$ & 4,81 & $\begin{array}{c}\text { Muy alto } \\
\text { cumplimiento }\end{array}$ \\
\hline & Total indicador: & $\mathbf{4 , 7 3}$ & $\begin{array}{l}\text { Muy alto } \\
\text { cumplimiento }\end{array}$ \\
\hline
\end{tabular}

Corresponde este resultado, de forma muy alta, a lo que describe Escudero (2013), cuando afirma que el propósito de planificar los productos no es otro que generar la estimación de ventas para la organización, es decir, qué fracción de la demanda será posible satisfacer con la producción de sus bienes y/o servicios. Por esta razón, contar con un sistema efectivo de planificación de productos, permitirá a la organización proyectar eficientemente sus actividades y procesos, cumpliendo con sus presupuestos de ingresos y de sus costos, sin perder de vista sus políticas y presupuestos, alcanzando, de esta forma, sus objetivos estratégicos.

De igual manera se validó la posición del investigador, para quien la planificación de los productos es una actividad clave dentro de las actividades de apoyo al proceso logístico, en ella descansa la razón de ser de toda logística empresarial. A este respecto, las organizaciones deben cambiar el foco de contener sus costos, por el de agregar valor, y en esta línea están todas las actividades relacionadas con la inversión y desarrollo de sistemas de planificación o pronósticos de la producción y ventas.

La tabla 6 presenta los resultados obtenidos para el último indicador utilizado para medir las actividades de apoyo de la gestión logística, denominado tratamiento de mercancías, en esta se aprecia un valor para la media de 4,67 indicando muy alto cumplimiento. Observándose en tal sentido que se realiza con muy alto cumplimiento todas las actividades medidas por los reactivos aplicados.

Tabla 6. Indicador: Tratamiento de mercancías

\begin{tabular}{lrcc}
\hline \multicolumn{1}{c}{ Ítem } & $\overline{\boldsymbol{X}}$ & Categoría \\
\hline 13. El tratamiento de mercancías apoya los objetivos de la producción. & 4,63 & $\begin{array}{c}\text { Muy alto } \\
\text { cumplimiento }\end{array}$ \\
$\begin{array}{l}\text { 14. El tratamiento de mercancías, dentro del proceso logístico, } \\
\text { constituye una de las actividades de apoyo para el eficiente } \\
\text { desarrollo de este proceso. }\end{array}$ & 4,88 & $\begin{array}{c}\text { Muy alto } \\
\text { cumplimiento }\end{array}$ \\
$\begin{array}{l}\text { 15. El sistema de flujo de mercancías apoya los objetivos de la recepción. } \\
\text { Total indicador: }\end{array}$ & 4,50 & $\begin{array}{c}\text { Muy alto } \\
\text { cumplimiento }\end{array}$ \\
\hline
\end{tabular}


De tal forma, se categorizo con muy alto cumplimiento el que: el tratamiento de mercancías apoye los objetivos de la producción $(4,63), y$, dentro del proceso logístico, constituye una de las actividades de apoyo para el eficiente desarrollo de este proceso $(4,88)$, así como también el sistema de flujo de mercancías apoye los objetivos de la recepción. $(4,50)$

Este resultado se relacionó, en muy alta medida, con lo expresado por Ballou (2004), para quien existen aspectos muy importantes del manejo de materiales, además de la geometría y herramientas, que incluye el movimiento de hombres, máquinas, herramientas e información. El sistema de flujo debe de apoyar los objetivos de la recepción, selección, inspección, inventario, contabilidad, empaque, ensamble y otras funciones de producción, constituyéndose en una de las actividades de apoyo básica para el eficiente desarrollo del proceso logístico.

Asimismo, coinciden muy altamente con la posición del investigador, cuando afirma que se debe considerar que esta actividad se relaciona con el proceso logístico en cuanto a las funciones primordiales que desarrolla como la selección del equipo, procedimientos de preparación de pedidos, almacenamiento y recuperación de mercancías; siendo eje medular para las empresas de bebidas bajo estudio, dado que es una de las actividades con mayor frecuencia de utilización.

La tabla 7 resume los resultados de la dimensión actividades de apoyo, con sus respectivos indicadores. En ésta, se aprecia que el valor para la media se ubicó en 4,50 indicando muy alto cumplimiento de las actividades de apoyo de la gestión logística en las empresas estudiadas. Este resultado es producto del nivel de cumplimiento alcanzado para cada uno de sus indicadores. De forma tal, se evidencia muy alto cumplimiento en lo relacionado a los indicadores compras $(4,54)$, almacenaje $(2,68)$, planificación de productos $(4,73)$, y tratamiento de mercancías $(4,67)$. Acotando que el indicador embalaje $(3,81)$ mostró alto cumplimiento.

Tabla 7. Dimensión: Actividades de apoyo

\begin{tabular}{lcl}
\hline SUB-DIMENSIÓN & $\overline{\boldsymbol{X}}$ & CATEGORÍAS \\
\hline Compras & 4,54 & Muy alto cumplimiento \\
Embalaje & 4,77 & Muy alto cumplimiento \\
Almacenaje & 3,81 & Alto cumplimiento \\
Planificación de productos & 4,73 & Muy alto cumplimiento \\
Tratamiento de mercancías & 4,67 & Muy alto cumplimiento \\
\hline \multicolumn{1}{c}{ Resumen Dimensión } & $\mathbf{4 , 5 0}$ & Muy alto cumplimiento del proceso \\
\hline
\end{tabular}

Estos resultados permitieron relacionar, de forma muy alta, lo que argumentan Cuervo y Osorio (2006), quienes indican que, en los procesos logísticos, las actividades de apoyo son el conjunto de actividades que se realizan para mantener en debida forma las instalaciones físicas y tecnológicas de la empresa en donde se llevan a cabo las demás actividades. Con frecuencia, el apoyo que proporciona a ésta para sus productos 0 servicios es tan importante para los clientes como la calidad del producto o servicio. 
De igual forma, validaron altamente lo expuesto por Anaya (2014) y Fontalvo y Vergara (2010); el primero, quien considera que estas actividades apoyan a las fundamentales o clave para que estas se cumplan, y generan los recursos que precisan las demás actividades, incluyendo: compras, embalaje, planificación de productos y tratamiento de mercancías, entre otras. De manera similar, los segundos las definen como actividades de soporte del sistema logístico, y se encuentran en las interfaces entre la logística, la producción y la comercialización, e incluyen: almacenamiento, manejo de mercancías, compra, planificación del producto, empaque, entre otras.

Visto estos resultados, el investigador deduce que en las empresas comercializadoras de bebidas en la Costa Oriental del Lago, se tiene muy alto valor de la importancia que poseen las actividades de apoyo dentro del proceso logístico que deben ejecutar en su gestión logística, lo cual se denota en el muy alto cumplimiento que se le dan a éstas como apoyo a las actividades fundamentales del proceso logístico, aportándoles los recursos necesarios para que puedan ser ejecutadas.

\section{CONCLUSIONES}

En lo concerniente al objetivo, donde se analizaron las actividades de apoyo de la gestión logística en las empresas comercializadoras de bebidas de la Costa Oriental del Lago, se concluye que estas organizaciones poseen muy alto cumplimiento de las actividades de apoyo, tomadas como aspectos fundamentales para describir la forma como se cumple el proceso logístico en las empresas señaladas.

Sin embargo, debe mencionarse que aun con esta categoría de muy alto cumplimiento de las actividades de apoyo, se detectó la existencia de un indicador, reseñado con alto cumplimiento, y no así con muy alto, en específico en las actividades de apoyo el almacenaje; asimismo, hubo indicadores tratados con muy alto cumplimiento que denotaron actividades (ítems) con alto cumplimiento, y no muy alto. Estos aspectos deben ser revisados por la gerencia, en virtud de la importancia que tiene la gestión logística.

\section{REFERENCIAS}

Anaya, J. (2014). El diagnóstico logístico: Una metodología para promover mejoras competitivas. Libros profesionales de empresa. Libros profesionales de empresa. Editorial ESIC. Madrid. España

Ballou, R. (2004). Logística. Administración de la cadena de suministro. Quinta Edición. Editorial Pearson Educación. México

Butter, F.y Linse, K. (2008). Rethinking procurement in the era of globalization. MIT Sloan Management Review. Pp. 7680

Cuervo, J. y Osorio, J. (2006). Costeo basados en actividades $A B C$ : gestión basada en Actividades. Tercera Edición. Deusto ediciones. Madrid. España

Escudero, M. (2013). Gestión logística y comercial. LOE: Administración y gestión. Primera Edición. Editorial Paraninfo S.A. Madrid. España

Fontalvo T. y Vergara J. (2010). La gestión de la calidad en los servicios. ISO 9001:2008. Primera edición. Editorial EUMED. España

La Cruz, W. (2010). La logística como herramienta para la competitividad en las empresas del sector agroindustrial. Tesis Doctoral. Universidad Rafael Belloso Chacín. Doctorado en Ciencias Gerenciales. Maracaibo, Venezuela

Pulido, J. (2009). Gestión de inventarios. El secreto de la rentabilidad. Primera Edición. Editorial Torino. Caracas. Venezuela 